\title{
Surgical treatment of colorectal cancer liver metastases: individualized comprehensive treatment makes a difference
}

\author{
Zhiwen Luo, Xinyu Bi \\ Department of Hepatobiliary Surgery, National Cancer Center/National Clinical Research Center for Cancer/Cancer Hospital, Chinese Academy of \\ Medical Sciences and Peking Union Medical College, Beijing, China \\ Correspondence to: Prof. Xinyu Bi, MD, PhD. Department of Hepatobiliary Surgery, National Cancer Center/National Clinical Research Center for \\ Cancer/Cancer Hospital, Chinese Academy of Medical Sciences and Peking Union Medical College, Beijing 100021, China. Email: beexy1971@163.com. \\ Comment on: Lillemoe HA, Vauthey JN. Surgical approach to synchronous colorectal liver metastases: staged, combined, or reverse strategy. Hepatobiliary \\ Surg Nutr 2020;9:25-34.
}

Submitted Oct 22, 2021. Accepted for publication Nov 22, 2021.

doi: 10.21037/hbsn-2021-23

View this article at: https://dx.doi.org/10.21037/hbsn-2021-23

The liver is the most common metastatic site for patients with colorectal cancer (CRC), and over $50 \%$ of cases show liver metastases. Furthermore, approximately $15-25 \%$ of CRC patients present with liver metastases at the time of diagnosis, and liver metastases are the primary cause of death in CRC patients. Although they lacked results from large-sample, prospective clinical trials, retrospective studies in the 1990s showed that hepatectomy is associated with long-term survival in patients with CRC liver metastases (CRLM), who benefited from a 5-year survival rate of more than $50 \%(1,2)$. However, the 5 -year survival rate of inoperable patients is less than $10 \%$. Despite there being broad consensus concerning the role of liver metastases resection several related issues remained to be examined further, especially those regarding synchronous colorectal liver metastases (SCRLM) and whether simultaneous or traditional staged resection is suitable. Concerning the staged resection process, the choice of which to resect first, the primary tumor or the liver metastases, is still controversial. Furthermore, issue of how to administer chemotherapy appropriately in the perioperative period remains unresolved.

In the 2020 issue of Hepatobiliary Surgery and Nutrition $(H B S N)$, Lillemoe et al. (3) comprehensively analyzed and compared the pros and cons of 3 commonly used surgical strategies for SCRLM: the traditional staged approach, the combined approach, and the liver-first approach. The traditional treatment algorithm for SCRLM is a staged approach that includes primary tumor resection followed by systemic chemotherapy and finally resection of liver metastases. The combined approach to SCRLM involves complete surgical resection of liver metastases at the time of primary colorectal resection. The liver-first or reverse strategy, the most recently proposed treatment for SCRLM, involves preoperative chemotherapy before hepatectomy followed by the later resection of the primary colorectal tumor. In the past, the traditional staged approach, in which the primary tumor is resected first, was advocated to prevent the emergence of new metastases and to reduce the risk of future complications of colonic obstruction, bleeding, or perforation. However, in patients with CRLM, liver metastases are the prime determinant of a fatal prognosis, and initial resection of the primary tumor might promote metastatic progression. In this regard, the combined approach, which involves complete surgical resection of liver metastases at the time of primary colorectal tumor resection, could prevent this problem but may increase the surgical risk. Therefore, it is necessary to carefully select suitable patients for this procedure. The liver-first approach initially resects the liver metastases, the main determinant in CRLM prognosis, while avoiding increasing the complication risk from a 2-site surgery. It is thus more suitable for patients with rectal cancer liver metastases who have a possible need for radiotherapy. In these patients, initial resection of liver metastases, followed by systemic chemotherapy plus local rectal radiotherapy, and concluded by primary rectal loci resection, is recommended.

Over recent years, perioperative management and 
surgical technology, especially minimally invasive techniques like laparoscopy, have been continually improving. Primary cancer and metastases sites can be resected by laparoscopy, which dramatically reduces the rates of surgical injury and complication. For instance, in our center, there is no significant difference in the complication risk or overall survival between the traditional staged approach and the combined approach $(4,5)$. However, the combined approach can reduce the hospital stay and costs. Hence, we are increasingly more willing to adopt the combined approach to manage CRLM. For patients with more liver metastases or those requiring large-scale liver resection or complex liver resection, the liver-first approach can also be used; consequently, use of the traditional staged approach has declined, as is indicated in the literature. The choice of surgical procedure needs to be decided upon according to several factors, including the patient's general condition, the size and number of liver metastases, the expected surgical extent, and comorbidities, such as primary tumor obstruction or perforation. Meanwhile, the tumor's biological behavior and its response to systemic therapy should also be considered when an individualized strategy is formed $(6,7)$.

At present, active surgical resection is a recognized concept in the management of CRLM. Surgical treatment should be actively carried out for operable patients. It is also necessary for inoperable patients to be provided an opportunity for active conversion therapy or for them to receive local treatment opportunities through surgery plus radiofrequency ablation or radiotherapy, among other methods (8-10). For patients with anticipated insufficient future liver remnant (FLR) volume, techniques like the portal vein embolization (PVE) or associating liver partition and portal vein ligation for staged hepatectomy (ALPPS) could help increase FLR and make resection opportunities available. However, surgeons should be acutely aware that the change in the concept of surgical treatment should not be attributed to the advancement of surgical technology but rather to the in-depth understanding of CRLM's biological behavior and the progress of systemic therapy. We now know that CRLM has an intermediate state between localized and disseminated disease, which is referred to as oligometastases (11). In this state, active surgery-based local treatment can confer better long-term survival to patients. However, we need to clarify that only when systemic therapy is effective can surgery resection, as a representative of local treatment, make a real difference. Therefore, emphasizing the relationship between the local and the systemic condition should be fully considered in the treatment of CRLM. For oligometastases, active surgery resection or surgery plus other local treatment should be encouraged; meanwhile, for a systemically metastasized disease, chemotherapy, as a representative of systemic therapy, should be the mainstay. We should also realize that the relationship between a localized and systemically metastasized disease is dynamic and changing. The oligometastases can progress to a state of widespread dissemination, but this can also be downgraded to an oligometastatic state through effective systemic therapy. Therefore, during the treatment process, continuous disease evaluation and timely treatment adjustment should be conducted. Additionally, a high-level multidisciplinary team (MDT) is the best way for patients to obtain accurate disease evaluation and a suitable treatment strategy (12). An MDT plays a pivotal role in making an accurate preoperative evaluation, deciding upon a comprehensive treatment strategy, realizing the optimal time for operation, and evaluating the therapeutic efficacy. Devising an individualized comprehensive treatment strategy for patients through an MDT is the key to achieving the longterm survival of CRLM patients.

\section{Acknowledgments}

Funding: This work was supported by Beijing CSCO Clinical Oncology Research Foundation CSCO-Linghang Project (Y-2019AZMS-0082) and the Capital's Funds for Health Improvement and Research (2018-1-4021).

\section{Footnote}

Provenance and Peer Review: This article was commissioned by the editorial office, Hepatobiliary Surgery and Nutrition. The article did not undergo external peer review.

Conflicts of Interest: Both authors have completed the ICMJE uniform disclosure form (available at https://hbsn. amegroups.com/article/view/10.21037/hbsn-2021-23/coif). The authors have no conflicts of interest to declare.

Ethical Statement: The authors are accountable for all aspects of the work in ensuring that questions related to the accuracy or integrity of any part of the work are appropriately investigated and resolved.

Open Access Statement: This is an Open Access article 
distributed in accordance with the Creative Commons Attribution-NonCommercial-NoDerivs 4.0 International License (CC BY-NC-ND 4.0), which permits the noncommercial replication and distribution of the article with the strict proviso that no changes or edits are made and the original work is properly cited (including links to both the formal publication through the relevant DOI and the license). See: https://creativecommons.org/licenses/by-nc-nd/4.0/.

\section{References}

1. Raoof M, Haye S, Ituarte PHG, et al. Liver Resection Improves Survival in Colorectal Cancer Patients: Causaleffects From Population-level Instrumental Variable Analysis. Ann Surg 2019;270:692-700.

2. Rees M, Tekkis PP, Welsh FK, et al. Evaluation of longterm survival after hepatic resection for metastatic colorectal cancer: a multifactorial model of 929 patients. Ann Surg 2008;247:125-35.

3. Lillemoe HA, Vauthey JN. Surgical approach to synchronous colorectal liver metastases: staged, combined, or reverse strategy. Hepatobiliary Surg Nutr 2020;9:25-34.

4. Bi X, Li C, Yan T, et al. Effect of surgical treatment on colorectal cancer with liver metastasis an analysis of 123 patients. Chinese Journal of Practical Surgery 2012;32:133-5.

5. Chen Q, Mao R, Zhao J, et al. Upgraded nomograms for the prediction of complications and survival in patients

Cite this article as: Luo Z, Bi X. Surgical treatment of colorectal cancer liver metastases: individualized comprehensive treatment makes a difference. HepatoBiliary Surg Nutr 2021;10(6):899-901. doi: 10.21037/hbsn-2021-23 with colorectal liver metastases treated with neoadjuvant chemotherapy followed by hepatic resection. Ann Transl Med 2021;9:265.

6. Fong Y, Fortner J, Sun RL, et al. Clinical score for predicting recurrence after hepatic resection for metastatic colorectal cancer: analysis of 1001 consecutive cases. Ann Surg 1999;230:309-18; discussion 318-21.

7. Luo Z, Chen X, Zhang Y, et al. Development of a Metastasis-Related Immune Prognostic Model of Metastatic Colorectal Cancer and Its Usefulness to Immunotherapy. Front Cell Dev Biol 2021;8:577125.

8. Abdalla EK, Vauthey JN, Ellis LM, et al. Recurrence and outcomes following hepatic resection, radiofrequency ablation, and combined resection/ablation for colorectal liver metastases. Ann Surg 2004;239:818-25; discussion 825-7.

9. Wong SL, Mangu PB, Choti MA, et al. American Society of Clinical Oncology 2009 clinical evidence review on radiofrequency ablation of hepatic metastases from colorectal cancer. J Clin Oncol 2010;28:493-508.

10. Takahashi H, Berber E. Role of thermal ablation in the management of colorectal liver metastasis. Hepatobiliary Surg Nutr 2020;9:49-58.

11. Hellman S, Weichselbaum RR. Oligometastases. J Clin Oncol 1995;13:8-10.

12. Li X, Chen Q, Bi X, et al. Effects of multidisciplinary team on the outcomes of colorectal cancer patients with liver metastases. Ann Palliat Med 2020;9:2741-8. 